\title{
Comparative Study on Prion Protein Detection Methods Using Biosensor Technology for Beef and Its Edible Products
}

\author{
Kazi Naziba Tahsin*, Rakib Hasan, Shahriar Khan \\ Department of Electrical and Electronics Engineering, Independent University, Bangladesh, Dhaka, Bangladesh \\ Email address: \\ naziba@iub.edu.bd (K. N. Tahsin) \\ ${ }^{*}$ Corresponding author
}

To cite this article:

Kazi Naziba Tahsin, Rakib Hasan, Shahriar Khan. Comparative Study on Prion Protein Detection Methods Using Biosensor Technology for Beef and Its Edible Products. American Journal of Biomedical and Life Sciences. Vol. 6, No. 1, 2018, pp. 1-8. doi: $10.11648 /$ j.ajbls.20180601.11

Received: November 23, 2017; Accepted: November 29, 2017; Published: January 17, 2018

\begin{abstract}
Prion diseases in humans are closely linked to consumption of bovine meant or organs infected with abnormal prions. Bovine organs which are most prone to prion disorders such as the brain and the liver, kidney etc are considered in various traditional cuisines over the world. Meat or edible parts should be tested safe from prions before releasing it into the market. The detection of prions proteins in bovine meat and other organs is a challenging task. The renowned methods include Quartz Crystal Microbalance and Surface Plasmon Resonance techniques. This paper narrates a performance analysis of the accuracy, sensitivity and feasibility of each technique over the other for the detection of abnormal prion protein. Successful detection of the infected prions will reduce the diseases related to abnormal prions in entering the food chain.
\end{abstract}

Keywords: Abnormal Prion Protein, Bioassay, Surface Plasmon Resonance Sensor, Quartz Crystal Microbalance

\section{Introduction}

Creutzfeldt-Jakob disease is the human form of Bovine Spongiform Encephalopathy. CJD is a neuro-degenerative disease caused by the misfolding of proteins. Misfolding of proteins, called prions, are the cause of the continuous death of neurons in the brain which eventually leads to the destruction of the brain and then death of the host. As of 2015, Public Health Agency of Canada published a report identifying 279 human cases of Creutzfeldt-Jakob Disease, inside Ontario and 776 human cases in Canada altogether. Amongst the different variations of CJD, variant CJD, is closely linked to the consumption of bovine meat contaminated with infected prions. There were cases of variant CJD observed in both 2014 and 2013 according to the fact sheet [1]. So the detection of prion proteins in meat or edible cow parts have become a prime research topic recently. Atempts to develop sensors which detect prions by binding to micro films that change when prions are present have been going on. This paper portraits the available techniques and their performance based on pion detection.

\subsection{Role of Prions}

Prion diseases are mostly sporadic but can also be infectious or inherited. Neurodegenerative diseases are caused when the normal TP PrP-C is replaced by a proteaseresistant. Naturally occurring PrP-C is situated usually in the brain and the modification of this protein results in abnormal neurobehavioral changes. Table 1 shows the properties of PrP-C. The conversion of $\alpha$ - helices of PrP-C into $\beta$ sheets is the basis of the formation of $\mathrm{PrP}-\mathrm{Sc}$ involves. As discussed below, the difference in the structure of PrP-C and PrP-Sc is one of the evidence that the fundamental event in the formation of infectious PrP-Sc is propagated from the conversion of $\alpha$-helices [3]. Renaturation experiments by Riesner, 2003 found that the $\beta$-sheet conversion is linked with the most stable state, during acidic $\mathrm{pH}$, is the $\beta$ - sheetrich aggregated state. It was reported that acidic and reducing conditions may induce a $\beta$-sheet-rich. Table 2 compares the properties of the infected $\mathrm{PrP}-\mathrm{Sc}$ and uninfected PrP-C. 


\subsection{Effects of Prions in the Body}

Till date, human prion diseases are Creutzfeldt-Jakob disease (CJD), Gerstmann-Str"aussler syndrome (GSS) and kuru. These diseases are recently shifting towards global concerns. The property of the transmissible agent or the prion itself is a research topic and the arising fears that through dietary exposure to infected tissues could bring an epidemic of a newly recognized bovine prion disease in public health. The simplest route for the contamination with prion proteins into the human body is through the food chain. The consumption of meat infected prion is at first absorbed into fatty tissues in the digestive tract such as the stomach glands. The properties of the protein are so hydrophobic that it would easily enter the immune system and to the blood circulation system. Concentrated in the spleen, inside the micelle environments, the protein is protected well from aqueous exteriors. Finally targeting the myelin sheaths of the nerve fibers, due to having high fat contents, it will eventually reach the human brain. This usually takes up a time so inoculation period of these occurrences are 4 to 5 years [2]. After reaching the brain, the infected prions cause severe neurological disorders and is the reason of the sponginess of the brain after being infected. In order to ensure meat safe from infected prions the necessity to detect infectious prion protein in meat products is essential.

\subsection{Reasons for Detecting Prions}

\subsubsection{Prions in Food for Human Consumption}

Animal protein is an important part of the human diet. The meat before taken to the market should be tested free from prions. Although acquired prion disease rate are low, the occurrence of CJD in humans in UK was huge. This happened mainly due to eating beef or other ruminant meat previously infected from BSE.

Table 1. Properties of PrP-C.

\begin{tabular}{|c|c|}
\hline & Property of PrP-C \\
\hline Structure & $\begin{array}{l}\text { Glycolipid-anchored cell membrane syalio } \\
\text { glycoprotein with four a-helical domains and two } \\
\text { asparagine-linked carbohydrate side-chains. }\end{array}$ \\
\hline Molecularweight & $27,000 \pm 35,000$ \\
\hline Location & $\begin{array}{l}\text { Low amounts in glial cells of the CNS. Peripheral } \\
\text { cells. Neurons in the Brain \& spinal cord }\end{array}$ \\
\hline
\end{tabular}

During the UK outbreak, many public health preventive measures were suggested in 1996. The following list is a summary of the measures [15]:

a. BSE infected risk material prohibited for human consumption.

b. Cattle less than 30 months old are not used for human food.

The methods introduced in 1996 were set to demolish acquired human CJD by reducing BSE-contaminated meat from entering the human and other farm animal food supply system. In the SBO ban, certain tissues were eliminated from the human food chain. This included brain, spinal cord, tonsil, thymus, spleen and intestines. Imposing a ban on such parts causes social, cultural and economic pressures. Juvenile cow brains are parts of traditional cuisines of European countries named sesos and are eaten in tacos and quesadillas. Many countries in South Asia cook the dish Maghaz which constitutes of fried ruminant brain. Veal is a popular ingredient in Italian and French cuisine Veal cutlets, such Italian cotoletta or Austrian Wiener Schnitzel are popular worldwide. Veal Parmigiana is a Italian-American dish of crumbed veal cutlets. Bones of calves are a source of stock for sauces and soups such as demi-glace. Cheese production uses rennet originating from calf stomachs. Many consumers are unaware of the SBO ban and still consume such animal proteins in their diet. There is still a question of how effective the SBO ban is worldwide and how strictly it is followed. Therefore, BSE still continues to be detected, at a very low rates, in farms even after the methods had been implemented in 1996 [15]. The easier and better way could have been to randomly test meat or other parts from farm and introduce methods to remove prions from food. Detection methods need to be feasible and accurate.

\subsubsection{Prions in Pet and Farm Feed}

The main cause of BSE epidemic had been because of ruminant proteins feed to ruminants. The FDA has imposed a ban on feeding mammalian-derived proteins to cattle. Ruminant-derived protein is still used as feed in swine and poultry farms since they are thought to be susceptible from BSE infection. Mammalian blood and blood products; gelatin; meat products for humans; milk products; and avian/swine proteins are exempt from the ban. In 2004, FDA officials have declared they would expand the feed ban by prohibiting, from ruminant feeds, ruminant blood and blood products, and restaurant plate waste. Research suggests that the ban of additional products from feed are necessary, prion protein detection of such feed before market delivery is necessary.

Table 2. Properties of PrP-C and PrPSc compared and contrasted.

\begin{tabular}{lll}
\hline Property & Non infective PrP-C & Infective PrP-Sc \\
\hline Primary structure & Alpha helical & Alpha helical \\
Structural ratio & $40 \% \alpha$ - helical content, & $20 \% \alpha$ - helical content, \\
Reaction with & none/ little $\beta$-content. & $50 \% \beta$-content. \\
Water & Non hydrophobic & hydrophobic \\
\hline
\end{tabular}

\subsection{Limitations in Detecting Infected Prion Proteins}

Accuracy of designing biosensors for quantitative and sensitive detection requires an understanding of its properties which were explained before. The properties that make prion protein PrP-Sc makes it difficult to detect in tissue samples are as follows:

a. The primary structures of PrP-Sc and PrP- C are not only similar but are identical. The binding agents that show affinities for PrP - Sc also show affinity for PrP C [10].

b. $\operatorname{PrP}-\mathrm{Sc}$ is species specified and cross species detection has negative effects on the efficiency of the sensor. If detecting for prions responsible for human to bovine 
infections, it is more wise to use binding agents more compatible to human or bovine PrP Sc [4].

c. PrP Sc is buried deep in lipid layers.

d. Origination of the sample has effects on the concentration of the PrP- Sc in the sample. It is not necessary that meat from all body parts will show the same affinity for the prion.

e. Prions are highly resistant to inactivation by ionizing radiation, protease and several chemicals so making both, pre-treatment and post treatment, a complicated process [10].

\section{Techniques for Detecting Prions}

\subsection{Bioassay Sensor Technology}

The earliest methods of detecting PrP- Sc proteins is to observe the brain samples obtained post slaughter under the microscope [7]. Drawbacks of the traditional technique is that it is very labour intensive, expensive and time consuming [5]. It is difficult to use this technique to tissue samples other than the brain.

Development of this technique was to the staining of the
PrP-Sc with an antibody specific for that PrP molecule, and observing this through the microscope as shown below in [7]. This allowed for a better view and a more quantitative analysis. Since PrP - C and PrP- Sc are both present on the sample; a pre-treatment for destroying the normal $\mathrm{PrP}-\mathrm{C}$ is necessary.

An assay system is used for investigating or analytic procedure, in many fields, for qualitative or quantitative analysis of the presence of a target analyte. The analyte is usually a tissue from an animal or organism. The entity to be detected is usually called the analyte, or the target of the assay. A biosensor uses these principals, to detect a biological agent for recognition along with a physical transducer to convert the biological recognising element to an electrical impulse [14]. To overcome the second problem stated in Section 1.1 an array of bio- analytes that differ in their affinities and specificities for PrP-Sc and $\mathrm{PrP}-\mathrm{C}$ are used for detecting the infective prions in a sample of tissue. It is possible to look for PrP- Sc in a sample of tissue by using only specific binding for it but not binding to any other analytes, including $\operatorname{PrP}-\mathrm{C}$. Figure 1 shows a typical bioassay system used for PrP Sc detection.
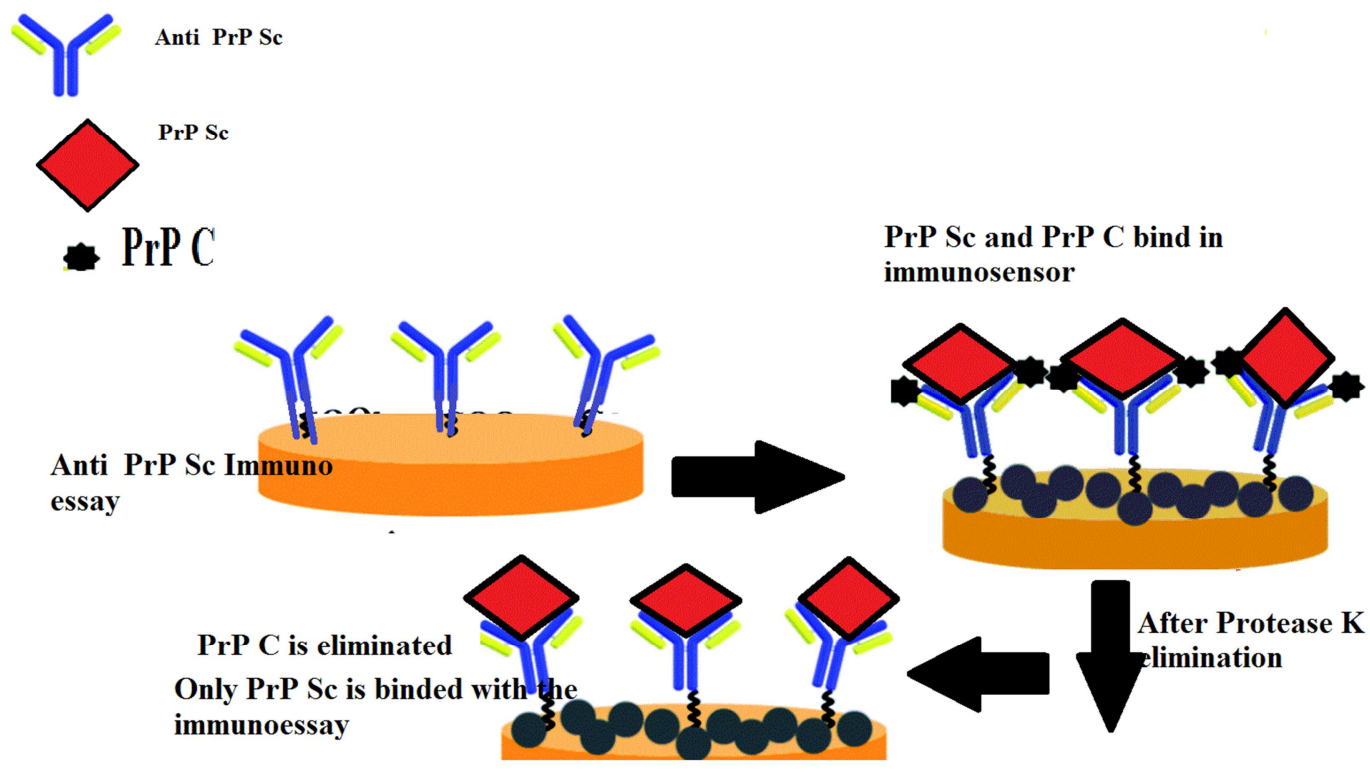

PrP Sc and PrP C bind in immunosensor

Figure 1. Bioassay using immono- sensing techniques.

There are several Bioassay techniques used in the industry of food and medicine industry. US Patent $6,165,784$ used the ELISA method where antibodies for the detection of PrP - Sc to determine the presence of prions in mixed samples. The Enzyme Linked Immuno-Sorbent Assay (ELISA) test is where an anti-PrP-Sc antibody, located on the sensor film, binds with the PrP- Sc. After washing, another enzyme-linked anti-PrP- Sc antibody binds to the attached PrP. The later enzyme linked anti PrPSc then catalyses a colour development reaction where the intensity of the colour is used for quantity measurements of PrP-Sc in the sample.

To increase the sensitivity of the test, remaining PrP- $\mathrm{C}$ is eliminated with a protease. However this elimination step has flaws like being complex, time consuming and expensive. This is also considered as less accurate as it dilutes the sample. The Platelia system uses this method of prion detction [1].

The Western Blot technique has improvised the former technique by denaturing PrP-Sc after the protease digestion, in order dissolvle and then further purify by gel electrophoresis. The dissolved PrP- Sc is then transferred to a test membrane and stained by an anti-PrP antibody attached to an indicating enzyme. This enzyme is responsible for a light emitting reaction which can be detected photographically. Although it has overcome the diluted 
sample problem, and is more sensitive, the protease $\mathrm{K}$ digestion is still not eliminated and the test is more time consuming than the ELISA method. The Prionics AG uses the western blot technique [4].

Although prion protein development is still developing, there still remains a need for a more sensitive assay system that is fast and feasible. This sensor would identify infected prions tissue from animals, would be sensitive enough for detection of low levels of prions present in the samples. In particular, an assay for the routine monitoring of meat samples would be useful to reduce the spread of the disease through the food chain as most of these animals are used for direct human consumption. An assay that can test suspected meat samples quickly could avoid the mass wastage of uninfected food in the food industry. There are several sensor systems that have been shown to respond to the binding and subsequent molecular changes of biological molecules. Throughout the years, several sensor systems have been developed, which to respond to the binding and subsequent molecular changes of biological molecules. The most common amongst them are the surface plasmon resonance (SPR) based immunosensors and the Thickness Shear Mode (TSM) acoustic devices for the detection of PrP- Sc and can be used in the meat industry using tissues.

\subsection{The Surface Plasmon Resonance Immuno - Sensor}

The Surface Plasmon Resonance uses the capable of detecting the refractive changes in a film when molecules bond to the sensor surface. The sensory system usually consists of an external light source such as a laser light source, an optical receptor module and an optical transducer chip. The laser source reflects light from one side of a thin metal film and sets up an electric field refracting on the other side of the metal film. The electric field extends into the bioassay sample, and the mass of the attached prion molecules cause changes in the field. This causes changes in the reflection angle of the light beam.

There are 3 types of SPR sensors used in the industry. Figure 2 shows the principal of each three SPR sensors: (a) prism coupler-based SPR system; (b) grating coupler-based SPR system; (c) optical waveguide-based SPR system [6]. The sensor sensitivity, stability and resolution depend upon properties of both the optical system and the transducing medium. The selectivity and response time of the sensor are primarily determined by the properties of the transducing medium. The sensitivity of each sensor is described in Figure 4 [18].

The abnormal prion protein biosensors must consist of a transducing medium which is an assay of biological agents which will only bind to the abnormal prion molecules [6]. In our application, the SPR biosensors includes antigenantibody method. The method of bioassay antibody binding using the ELISA assay is followed by binding an anti-PrP-Sc antibodies a surface exhibiting a protein affinity. a)

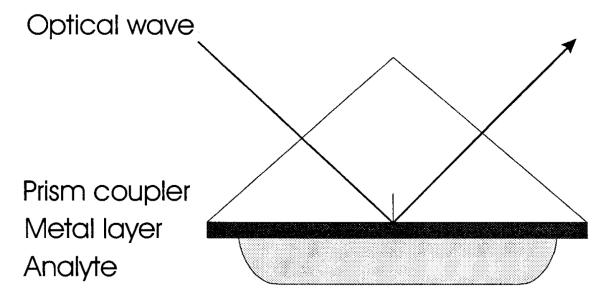

b)

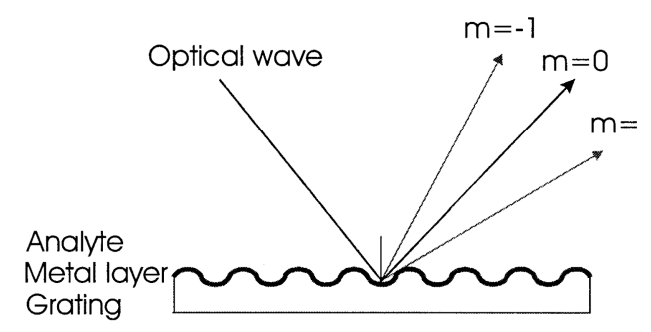

c)

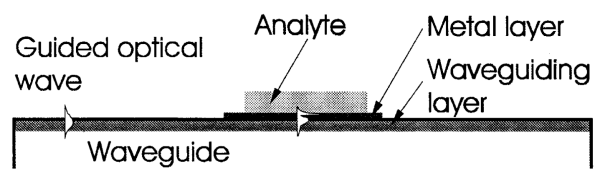

Figure 2. The types of SPR Sensors: Prism Coupled (a) \& Grating Coupler based (b) and optical wave based (c) Guided optical wave [6].

Sensitivity of a SPR biosensors is determined by differentiation of the observed SPR parameter, such as resonant angle or wavelength, by the parameter to be measured, usually the refractive index, or mass deposited or concentration. It can be seen from the Figure 4 that the sensitivity of a sensor increases with a decrease in wavelength according to angular interrogation. This is opposite for wavelength and intensity interrogation. It can concluded from [16] that for a given wavelength, the Prism coupled based SPR is more sensitive than the grating coupler based SPR. This is why for the PrP - Sc detector we have chosen the prism based approach.

During the homogenous binding of the assays, the detection takes place in the solution of the prion and is considered very slow detection process. Since the SPR is selective to surface bound changes in the analyte surface and occurs in very minute distances away from the immuno assays the choice of heterogeneous immuno sensors are selected for Prion protein detection. They are also known for better sensor signal intensities and rapid detection.

Resolution of a sensor cannot be altered much by external properties such as the above, and is dependent on the sensor conditions such as temperature, choice of light source and the noise of elimination method used by the light detector. The accuracy of biosensors depends on the experimental conditions and is found by the ratio of the assumed value or amount of the analyte present by the sensitivity of a particular detection approach.

The most researched topic is the question of which metal to be selected as the transduction surface as polarized light is refracted through the glass prism to the metal/solution 
dielectric interface. Research has been done over a wide range of incident angles which has an effect on the intensity of the resulting reflected light. The minimum angle responsible for reflectivity, known as the critical angle is also taken into account as the SPR angle.

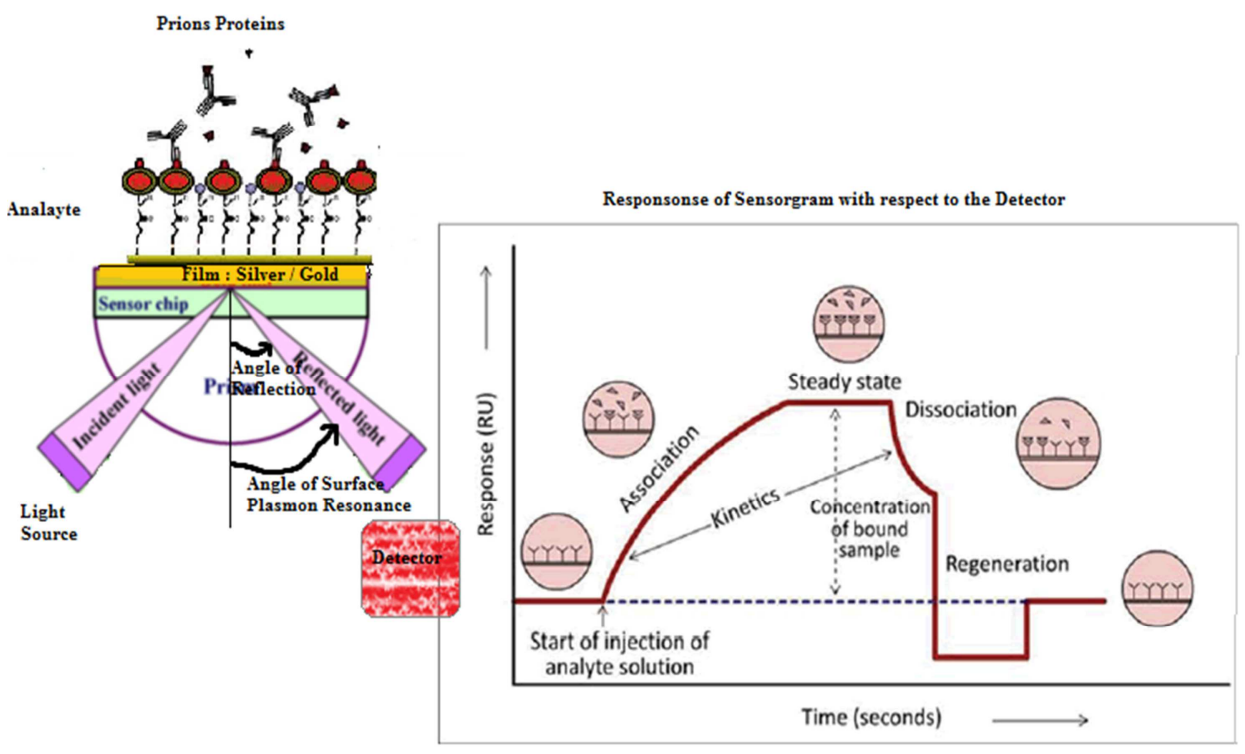

Figure 3. SPR operation and change in resonance with the binding of the analyte $(\operatorname{Pr} P-S c)$.

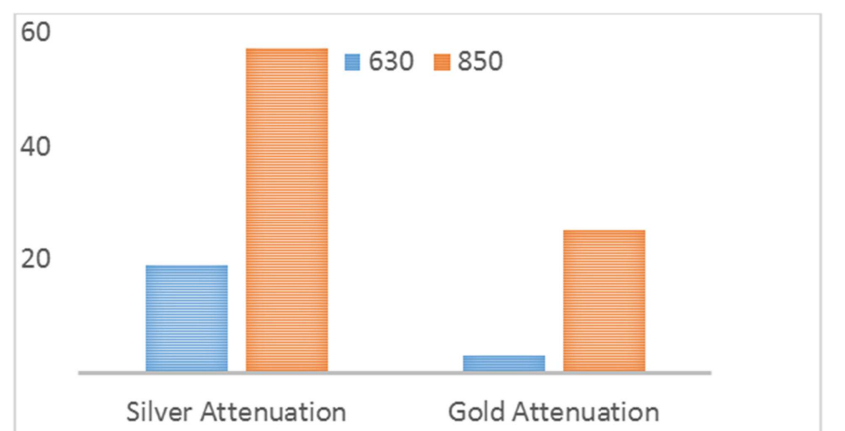

Figure 4. Attenuation of surface plasma waves (SPW) with different films [18].

The choice of gold - prion antigen as dielectric between biological agents such as prions is highly preferred due to its chemical stability and free electron behavior.

The sensors system described below is the prism coupler based SPR consisting of a layer of metal binding layer (gold) and a sensing layer (protein chip) attached to one side of the layer of metal (gold). The protein chips are used to bind large number of anti- PrP - Sc, immobilized to integrated photo microchip. Sometimes the immobilization of PrP-Sc may be done first and then exposed to the sensing surface layer. The sample may also be washed with protease $\mathrm{K}$, before exposing to the sensor surface, as protease treatment removes $\mathrm{PrP}-\mathrm{C}$ but not $\mathrm{PrP}-\mathrm{Sc}$. The sensing response occurs as $\mathrm{PrP}-\mathrm{Sc}$ is bound by anti- PrP Sc, or otherwise interact with, the sensing layer. The interaction of the PrP Sc/antibody with the sensing layer is monitored by an optical sensor located on the opposite side of the SPR sensor surface, which detects a change in the refraction and/or reflection of an optical beam as shown Figure 4.

\subsection{Transverse Shear Mode Acoustic Wave Sensor}

In the past decades, it was found that the resonant frequency of a quartz crystals changes not only to mass deposition, but also to coupling between the crystal and the analyte [5]. There are two types of acoustic wave sensors: surface acoustic wave (SAW) and quartz crystal microbalance (QCM).

Quartz Crystal Microbalance is a thickness shear mode (TSM) acoustic wave resonator, in which an AT-cut at an angle of 32.5 to the $\mathrm{z}$-axis is sandwiched between two metal electrodes. Saurbey has developed that if a layer of foreign mass rested on the plate, the decrease in frequency was proportional to the density of mass deposited as shown in the equation below:

$$
\Delta f=-\Delta m * \frac{2 f o^{2}}{A \sqrt{\tau \rho}}=S i \Delta m
$$

Patent WO 2006/063437 the sensing layer was composed of PrP-C on the TSM and the response of the acoustic sensor resonance was observed after detecting PrP-Sc in a given tissue sample. The response of the sensor occurs due to combination of $\operatorname{PrP}-\mathrm{C}$ and $\operatorname{PrP} \mathrm{Sc}$, converting the $\operatorname{PrP}-\mathrm{C}$ on the sensor to form new PrP Sc. Due to PrP-C being hydrophobic in nature as discussed in table 2, the sensor becomes more hydrophobic as time increases, therefore the resonant frequency increases continuously. The patent suggested the use of tissues such as brain and various bodily tissues. This makes TSE sensors appropriate for use in food testing of body parts and muscle tissues from cattle to be tested before consumption.

Sensitivity of the TSM depends on the resonance frequencies of these devices are usually in the range of 10-50 
$\mathrm{MHz}$ [9]. At higher frequencies, the mass sensitivity increases but the film becomes too thin. Experiments on the sensitivity depending on the frequency in [18] showed better results with waves of $50 \mathrm{MHz}$ than with $10 \mathrm{MHz}$ but with high attenuations of around $30 \mathrm{~dB}$.

The thickness of the electrode, decides the mass sensitivity quality factor of the TSE. Research carried out by Steinem, 2000 , suggested that if the thickness of the quartz electrode was too thin, the resonant frequencies acted as if the region was without the electrode and decreases the Q- factor of the device [16]. Dead dielectrics like gold, combined with high frequency resonators worsen the Q-factor. Usually Aluminium with a lower absorbing values are used. The radial mass sensitivity is dependent on the square of radial displacement. The highest sensitive region is around the center and decreases along the edges of the dielectric.

The paper by Poturnayova et al. 2014, discussed the immobilization of aptamers or monoclonal antibodies for detecting of PrP-C by electrochemical quartz crystal microbalance (EQCM) sensitive to $20 \mathrm{pM}$ for antibodies and $50 \mathrm{pM}$ for DNA aptamers [12]. Small amounts of surfactant tween $\mathrm{n}$ phosphorous buffers aided the binding of PrP-C to antibodies protein A (PrA) and antibodies (PRI 308). Experiments proved that the method could be used to detect pathological prions PrPSc, but the level of detail needs to be improved for PrP-Sc purpose and the application conditions of the sensor in real samples should be optimized.

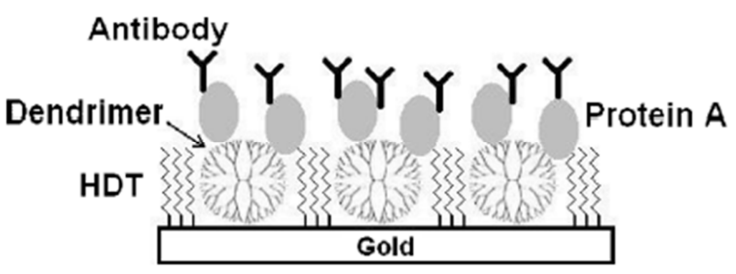

Figure 5. Immuno-quartz crystal microbalance [12].

\section{Performance Analysis of Techniques}

In 1995 Koblinger et el, set up comparisons of QCM and SPR in terms of sensitivity and cross reactivity [19]. Limitation arises due to their functional differences which effect their sensitivity, reproducibility, SNR, analyte compatibility and set up cost. The cost of set up of QCM is much lower than that of SPR as they use lasers or silicon chips. Bioanalytical measurements for affinities can be done accurately with both devices. QCM is much more suitable for detailed analysis of properties. SPR is much sensitive than the QCM as it detects more particles per unit area. The QCM occupies three times more space than the SPR. Table 3 compares and contrasts the special functions of both of the sensing techniques.

Table 3. Contrast properties of $Q C M \& S P R$.

\begin{tabular}{|c|c|c|}
\hline & SPR & QCM \\
\hline Wave characteristics & Surface plasmon & Mechanical shear \\
\hline Equations & Classical electrodynamics & Wave propagation in anisotropic medium \\
\hline Measurant & Critical angle & Q factor, resonant frequancy, impedance \\
\hline Sensitivity parameters & $\begin{array}{l}\text { For a } 5 \mathrm{MHz} C \mathrm{CQM} \text { can be } 20 \mathrm{ng} / \mathrm{cm}^{2} \text { per } \mathrm{Hz} \text {. } \\
\text { High frequency increases the sensitivity. }\end{array}$ & $\begin{array}{l}\text { Depends upon properties such as polarization of the prions, } \\
\text { wavelength of light, and prism index of refraction, typical values } \\
\text { are } 1 \mathrm{ng} / \mathrm{cm}^{2} \text { per mdeg. }\end{array}$ \\
\hline Penetration in fluids & $150 \mathrm{~nm}[19]$ & $126 \mathrm{~nm}[19]$ \\
\hline Ionisation effect & Ionization high & None \\
\hline Thickness of film & $0.0263 \mathrm{n} / \mathrm{m}$ & $184 \mathrm{~Hz} \mathrm{n} / \mathrm{m}$ \\
\hline Effective change noticed on sensor & $0.005^{\circ}$ & $20 \mathrm{~Hz}$ \\
\hline Crossectional area minimul & $0.005 \mathrm{~mm}^{2}$ & $5 \mathrm{~mm}^{2}$ \\
\hline Reusability & Yes and known as label free detectors & $\begin{array}{l}\text { No. Reusing QCM sensor would damp, drastically, degrading its } \\
\text { performance. }\end{array}$ \\
\hline Replicable & Yes & $\begin{array}{l}\text { Maintaining the Limit Of Detection along with reproducibility of } \\
\text { QCM measurements very challenging. }\end{array}$ \\
\hline
\end{tabular}

Both methods need to be submerged together to create an effective system for detection. Combining the quartz crystal microbalance with SPR will increase a double mode impedance study.

\section{Future Work on Detection}

Researchers are studying Surface Acoustic Wave devices which use the piezoelectric principal of wave generation. A surface acoustic wave (SAW) is an acoustic and mechanical wave that propagates from the piezoelectric cut. Usually, an electrical impulse is converted into polarized transversal waves travelling parallel to the surface of sensor, due to the piezoelectric properties of the substrate material.

This approach can used to detect prions as it is sensitive to biological analytes. The sensitivity of the SAW for surface modifications is increased, by the sensor material, and the design of the layer as well as by the structure of the sensor and the transducers the sensor is defined by the coating of the sensor surface with antibodies [18]. 
Table 4. Merits and demerits when using $Q C M$ \& SPR for Prions detection.

\begin{tabular}{|c|c|c|}
\hline & SPR & QCM \\
\hline Merits & $\begin{array}{l}\text { Reusability, lowering the cost of commercially usage. } \\
\text { Regeneration buffer can be used to disrupt the interaction between the } \\
\text { prion and antibody, meaning that the sensor (with the ligand attached) } \\
\text { can be reused. Ligand stability is dependent on the reusability factor. } \\
\text { SPR total mass LOD is } 1 \text { fg, which makes SPR more sensitive than } \\
\text { QCM. In order for QCM to to perform with same sensitivity level, the } \\
\text { area of the sensor should be larger than that of SPR sensors. The } \\
\text { space required was seen to be reduced in terms of SPR sensing. }\end{array}$ & $\begin{array}{l}\text { The flow-rate has to be adjusted to result in the same mass } \\
\text { transfer. The flow-rate of } 10 \mu 1 / \text { min in SPR corresponded to } 73 \\
\mu 1 / \text { min flow rate in QCM. So for comparable results, the flow-rate } \\
\text { used in the experiment should be } 7.3 \text { times higher for QCM than } \\
\text { for SPR. }\end{array}$ \\
\hline Demerits & $\begin{array}{l}\text { The flow rate was found } 7 \text { times lower than QCM. } \\
\text { Signal amplification was required as } 0.1 \% \text { of the surface receptors to } \\
\text { be occupied to detect their presence. } \\
\text { Prions immobilized onto SPR sensor surfaces sometimes loose their } \\
\text { bioactivity and, with some immobilization agents, only a small } \\
\text { portion of the immobilized biomolecules are active. }\end{array}$ & $\begin{array}{l}\text { High temperature causes deviations in measurements so } \\
\text { temperatures must be kept very stable. } \\
\text { Humidity causes a change in frequency on the QCMforming } \\
\text { micro droplets on the crystal adding extra mass. } \\
\text { Liquids cause surface inhomogeneity, disassociation of the antigen } \\
\text { prions or viscosity changes effecting the response in QCM. }\end{array}$ \\
\hline
\end{tabular}

\section{Conclusion}

Prion diseases are occurring in many places due to consuming animal proteins infected with prions. Many body parts of animals for consumption are banned under the SBO. However meat could be tested and several other parts could be consumed in delicate cuisines if proper detection methods were used. The SPR and the QCM techniques are the most widely used methods for prion detections till date. The SPR produced more accurate, sensitive results during the experiments with being reusable at the same time, concluding that it is a more reliable abnormal prion protein detector than the QCM technique which needs more modification for prion detection purposes. This paper represented the performance analysis on prion protein detection systems and biosensors available in the market.

\section{Acknowledgements}

Authors would like to thank the Food Science Department of the University of Guelph, Guelph, Ontario, Canada for their support and guide during this project and also the School of Engineering of University of Guelph for their resources.

\section{References}

[1] CJD CASES BY PROVINCE/TERRITORY, Available at:. www.publichealth.gc.ca.

[2] L., 2005. Thinking the unthinkable: Alzheimer's, CreutzfeldtJakob and Mad Cow disease: the age-related reemergence of virulent, foodborne, bovine tuberculosis or losing your mind for the sake of a shake or burger. Medical hypotheses, 64(4), pp. 699-705. Available at: http://www.ncbi.nlm.nih.gov/pubmed/15694685.

[3] Cohen, F. E. et al., 1993. Conversion of alpha-helices into beta-sheets features in the formation of the scrapie prion proteins. Proceedings of the National Academy of Sciences of the United States of America, 90(23), pp.10962-10966. http://www.pnas.org/content/90/23/10962.abstractln http://www.pnas.org/content/90/23/10962.full.pdf.
[4] Gordon Hayward, Warren Stiver, Jonathan Ellis, V.W., 2006. Prion Sensors for Diagnosis of Transmissible Spongiform Encephalopathy or for Detection of Prions, and Use Thereof. html. Available at:

http://www.google.com/patents/CA2589751A1?cl=en.

[5] Hayward, G. L. \& Thompson, M., 1998. A transverse shear model of a piezoelectric chemical sensor. Journal of Applied Physics, 83(4), pp.2194-2201. Available at: http://link.aip.org/link/?JAP/83/2194/1.

[6] Homola, J., Yee, S. S. \& Gauglitz, G., 1999. Surface plasmon resonance sensors: review. Sensors and Actuators B: Chemical, 54(1-2), pp.3-15.

[7] Ishikawa, K. et al., 2004. Amyloid imaging probes are useful for detection of prion plaques and treatment of transmissible spongiform encephalopathies. The Journal of general virology, 85(Pt 6), pp.1785-1790.

[8] Kraus, A., Groveman, B. R. \& Caughey, B., 2013. Prions and the Potential Transmissibility of Protein Misfolding Diseases*. Annual Review of Microbiology, 67(1), pp.543564. Available at: http://www.annualreviews.org/doi/abs/10.1146/annurevmicro-092412-155735

[9] Länge, K., Rapp, B. E. \& Rapp, M., 2008. Surface acoustic wave biosensors: A review. Analytical and Bioanalytical Chemistry, 391(5), pp. 1509-1519.

[10] Larry Green, 2004. Methods and compositions for detection of bovine spongiform encephalopathy and variant creutzfeldtjacob disease. Available at: http://www.google.com/patents/US20040018554.

[11] Ordal, M. a et al., 1983. Optical properties of the metals Al, $\mathrm{Co}, \mathrm{Cu}, \mathrm{Au}, \mathrm{Fe}, \mathrm{Pb}, \mathrm{Ni}, \mathrm{Pd}, \mathrm{Pt}, \mathrm{Ag}, \mathrm{Ti}$, and $\mathrm{W}$ in the infrared and far infrared. Applied optics, 22(7), pp.1099-1020.

[12] Poturnayova, A. et al., 2014. Comparative Analysis of Cellular Prion Detection by Mass-Sensitive Immunosensors., pp. 1312-1319.

[13] Riesner, D., 2003. Biochemistry and structure of $\operatorname{Pr} P C$ and $\operatorname{Pr} P$ Sc. British Medical Bulletin, 66(ii), pp.21-33.

[14] Shankaran, D. R., Gobi, K. V. \& Miura, N., 2007. Recent advancements in surface plasmon resonance immunosensors for detection of small molecules of biomedical, food and environmental interest. Sensors and Actuators, B: Chemical, 121, pp.158-177. [Accessed: 07- Nov- 2017]. 
[15] Smith, P. G. \& Bradley, R., 2003. Bovine spongiform encephalopathy (BSE) and its epidemiology. British Medical Bulletin, 66, pp.185-198.

[16] Steinem, C., 2000. Piezoelectric Mass-Sensing Devices as Biosensors-An Alternative to Optical Biosensors?

Angewandte Chemie (International ed. in English), 39(22), pp. 4004-4032. Available at:

http://www.ncbi.nlm.nih.gov/pubmed/11093194, Wilson, D.

A. \& Nixon, R. A., 2009. Sniffing out a function for prion proteins. Nature neuroscience, 12(1), pp.7-8. Available at: http://www.nature.com/neuro/journal/v12/n1/abs/nn01097.html \npapers3://publication/doi/10.1038/nn0109-7.
[17] Wohltjen, H. \& Roy, M. K., 1987. Surface Acoustic Wave Devices as Chemical Sensors in Liquids. Evidence Disputing the Importance of Rayleigh Wave Propagation. Anal. Chem., 59, pp.833-837.

[18] M. A. Ordal, L. L. Long, R. J. Bell, S. E. Bell, R. R. Bell, R. W. Alexander, J. Ward, C. A. Ward, optical properties of metals $\mathrm{Al}, \mathrm{Co}, \mathrm{Cu}, \mathrm{Au}, \mathrm{Fe}, \mathrm{Pb}, \mathrm{Ni}, \mathrm{Pd}, \mathrm{Pt}, \mathrm{Ag}, \mathrm{Ti}$, and $\mathrm{W}$ in the infrared and far infrared, Appl. Opt. 11 (1983) 1099-1119

[19] C. Koblinger, E. Uttenthaler, S. Drost, F. Aberl, H. Wolf, G. Brink,, A. Stanglmaier, E. Sackmann, Sens Actuators B. 1995, 24-25, 107. 